\title{
Attenuation Correction for the NIH ATLAS Small Animal PET Scanner
}

\author{
Rutao Yao, Member, IEEE, Jürgen Seidel, Jeih-San Liow, Member, IEEE, and Michael V. Green
}

\begin{abstract}
We evaluated an analytic attenuation correction method for the National Institutes of Health (NIH) Advanced Technology Laboratory Animal Scanner (ATLAS) small animal positron emission tomography (PET) scanner. This method is based on the body outline of emission images and an average empirical $\mu$ (linear attenuation coefficient) value. We evaluated this method using a computed tomography (CT)-based attenuation correction (ACs) as the "gold standard." A specially fabricated attenuation calibration phantom with cylindrical inserts that mimic different body tissues was used to derive the relationship to convert CT values to $\mu$ for PET. The methods were applied to three test data sets: 1) a uniform cylinder phantom, 2) the attenuation calibration phantom, and 3 ) a mouse injected with $\left[{ }^{18}\right.$ F]FDG. CT-based correction achieved activity restorations expected for all three data sets. The analytic method showed similar correction factors for the cylinder phantom and the mouse for which the attenuation medium volume matches the detectable emission source distribution. The correction factor for the attenuation calibration phantom was smaller because the emission image outline underestimated the attenuation medium volume.
\end{abstract}

Index Terms-Attenuation correction, computed tomography (CT), dual modality, imaging, positron emission tomography (PET), small animal imaging.

\section{INTRODUCTION}

A LTHOUGH the magnitude of attenuation in small animal PET is not as large as in human studies, $5 \mathrm{~cm}$ of soft tissue, the approximate transverse size of a $250 \mathrm{~g}$ rat, may absorb $40 \%$ of $511 \mathrm{keV}$ photons. Thus, attenuation correction (AC) is still potentially important for quantitatively accurate estimates of tracer concentration in high-resolution small animal PET. In this paper, we sought an AC protocol for the Advanced Technology Laboratory Animal Scanner (ATLAS) small animal PET scanner [1] at the National Institutes of Health (NIH), Bethesda, $\mathrm{MD}$, that is easily implemented for routine use, exhibits good accuracy, and adds minimal noise to the corrected emission images.

The AC methods developed for human PET scanners are applicable to small animal PET. The microPET animal PET scanners by Concorde Microsystems, Inc., Knoxville, TN, and the

Manuscript received November 15, 2003; revised June 14, 2004.

R. Yao is with the Department of Nuclear Medicine, State University of New York at Buffalo, Buffalo, NY 14214-3001 USA (e-mail: rutaoyao@buffalo.edu).

J. Seidel and M.V. Green are with the Department of Nuclear Medicine, Clinical Center, National Institutes of Health (NIH), Bethesda, MD 20852-1180 USA (e-mail: jurgen_seidel@ nih.gov; mgreen@cc.nih.gov

J.-S. Liow is with the Molecular Imaging Branch, National Institute of Mental Health, National Institutes of Health (NIH), Bethesda, MD 20852 USA (e-mail: liowj@intra.nimh.nih.gov).

Digital Object Identifier 10.1109/TNS.2005.851467
Mosaic animal PET scanner by Philips Medical Systems, Andover, MA, both use transmission sources for AC. However, methods based on transmission scans are disadvantageous because of added statistical noise and extra data acquisition time. Computed tomography (CT)-based AC, on the other hand, has the benefits of short scan time, low noise, and high spatial resolution [2], [3]. The main challenges in using CT methods are as follows:

1) CT attenuation factors need to be scaled up for use with PET data;

2) X-ray sources in CT emit photons with a spread of photon energies whereas PET images are formed from monoenergetic $511 \mathrm{keV}$ annihilation radiation;

3) $\mathrm{CT} \mathrm{AC}$ requires accurate spatial registration of the PET and $\mathrm{CT}$ images.

In addition to ATLAS, the Imaging Physics Laboratory in the Nuclear Medicine Department, Clinical Center, NIH, is equipped with a small animal CT scanner (microCAT II, ImTek, Inc., Knoxville, TN). It is possible, therefore, to implement an AC method based on the PET/CT method of Kinahan et al. [2]. However, since small animals have smaller ACs than humans, an analytic method that extracts the animal's body outline from the emission image and assigns an empirical $\mu$ to the body within that outline may also be sufficient to obtain a reasonable AC without an additional CT scan. In this paper, these CT and analytic methods are implemented and compared using the CT $\mathrm{AC}$ as the "gold standard."

\section{MATERIALS AND METHODS}

The ATLAS scanner has a $6.8-\mathrm{cm}$ transaxial field-of-view (FOV), a 2-cm axial FOV, and a central point source spatial resolution of $1.8 \mathrm{~mm}$ [full-width at half-maximum (FWHM)] [1]. All scans were acquired with a $250-700 \mathrm{keV}$ energy window. The central point source sensitivity for this energy window is $1.8 \%$.

\section{A. Phantom Studies}

Two phantoms were used for this study. The uniform cylinder phantom (Data Spectrum Corp., Hillsborough, NC) is $4.5 \mathrm{~cm}$ in diameter and $4 \mathrm{~cm}$ high. It can be filled with water or radioactive materials. A second phantom (Radiology Support Devices, Inc., Long Beach, CA), shown in Fig. 1, is a specially fabricated attenuation calibration phantom with three different cylindrical inserts (compartments A, B, and C) that mimic attenuation by spongiosa bone, cortical bone, and adipose tissue, respectively, and a fourth cylindrical chamber that can be filled with varying 


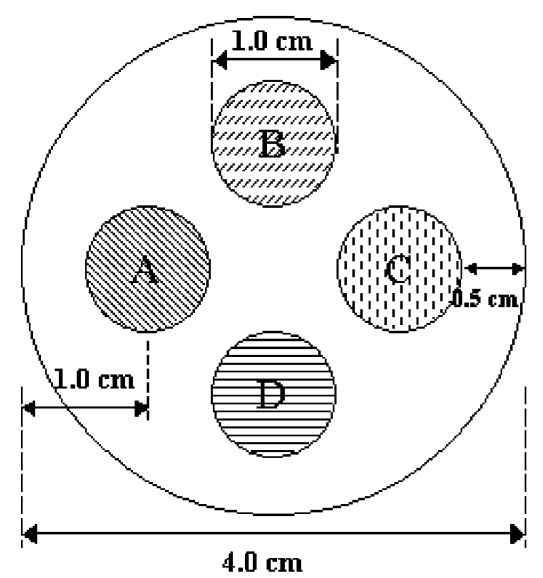

Fig. 1. End view of the attenuation calibration phantom. A, B, C, and D are small cylinder chambers with the same length as that of the phantom. A, B, and $\mathrm{C}$ are filled with artificial spongiosa bone, cortical bone, and adipose tissue, respectively. D can be filled for emission measurements.

concentrations of radioactivity (compartment D). The phantom is $4 \mathrm{~cm}$ in diameter and $10 \mathrm{~cm}$ in length.

\section{B. Mouse Study}

A mouse was injected with an IV with $500 \mu \mathrm{Ci}$ of $\left[{ }^{18} \mathrm{~F}\right] \mathrm{FDG}$. After a 45-minute uptake period, the animal was sacrificed and fixed with tape to the imaging bed of the ATLAS scanner. Approximately $5 \mathrm{~min}$ later, imaging began. The animal was stepped through the imaging FOV in six equal 10-min duration steps to obtain a near whole-body emission data set. At each step, an emission image was acquired such that consecutive scans overlapped each other by $4.5 \mathrm{~mm}$. Images at each bed position were reconstructed separately as described in the section hereafter and stitched together by assigning different weight factors to the overlapped slices.

Following the PET scan, the animal and bed were manually transferred to the CT scanner. CT images were acquired in all cases (phantoms and animal) with the X-ray tube high voltage set at $55 \mathrm{kVp}$ (with ImTek-provided Al filtration), 500 $\mu \mathrm{A}$ current, $500 \mathrm{~ms}$ exposure time, and 360 projections. The $\mathrm{X}$-ray tube's high voltage setting was chosen according to the method described in [4], i.e., optimal image contrast and resolution are achieved when the effective energy of the X-rays for which $\mu=2 / D$, where $D$ is the diameter of the subject and the effective energy is generally near $30 \%$ to $40 \%$ of the peak energy. For a 3-cm-diameter (mouse-sized) object, the optimal effective energy is approximately $25 \mathrm{keV}$. Considering the $\mathrm{Al}$ filtration increases the effective energy, e.g., to $50 \%$ of peak energy, $55 \mathrm{kVp}$ is about the optimized $\mathrm{X}$-ray voltage for mouse imaging.

All emission studies, phantom or animal, were performed in an activity/count rate regime where random coincidences were negligible.

\section{Reconstruction}

PET images were reconstructed with a three-dimensional ordered subset expectation maximization (3D-OSEM) exact positioning algorithm [5] running on an SGI Linux cluster of parallel computers. For the attenuation calibration phantom and the
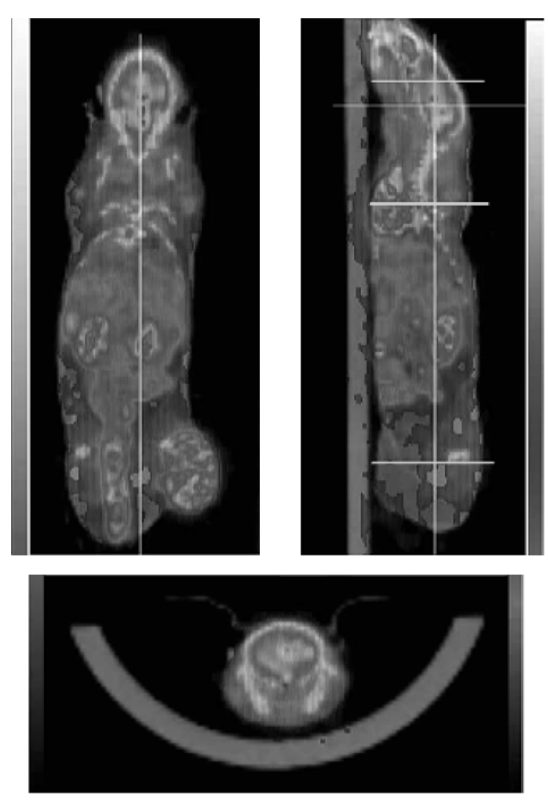

Fig. 2. Spatially registered and superimposed coronal (upper left), sagittal (upper right), and transverse (lower) view CT and ATLAS FDG PET images of the mouse. The gray scale bar on the left side is for the CT images and the one on the right side is for the PET image. The wider horizontal line in the sagittal view and the two vertical lines indicate the location of the sections. The three short horizontal lines in the sagittal view show the locations of the transverse images (head, heart, and tumor) in Fig. 7(a).

mouse studies, six iterations with eight subsets and four iterations with nine subsets were used, respectively. For the uniform phantom, one iteration with nine subsets was used. These parameters were selected based on the noise-resolution performance curve reported in [5]. The voxel dimensions in the reconstructed images were $0.5625 \mathrm{~mm} \times 0.5625 \mathrm{~mm} \times 1.125 \mathrm{~mm}$. CT images were reconstructed into $0.225 \mathrm{~mm}$ cubic voxels with a Shepp-Logan filter.

\section{CT and PET Image Registration}

For the phantom images, the registration of PET and CT images was done by aligning cylinder centers in the images. For the mouse study, a whole body PET image was obtained by combining the six bed position images and was registered with the CT whole body image. We assumed that the animal's position on the imaging bed did not change when the animal was moved from the PET scanner to the CT scanner at the end of emission imaging so that the PET and CT images could be spatially registered by simply shifting one data set relative to the other. No deformation or rotational adjustments appeared necessary by visual inspection of the fused images (Fig. 2); this was also confirmed using a commercial registration software (Nuclear Diagnostics Ltd., Hermes Multi-Modality Fusion Software, Northfleet, Kent, U.K.).

\section{E. Calculation of $\mu$ for PET From CT Data}

We used the attenuation calibration phantom to derive the relationship between $\mathrm{CT}$ value and the corresponding $\mu$ value at $511 \mathrm{keV}$ to be used for PET AC. A long transmission scan (6 h, 750 million counts) and a long blank scan (12 h, 1.6 billion counts) using a 68-Ge ring source (Sanders Medical Products, 
TABLE I

CT Image Segment Peak Values and the CorResponding $\mu$ Values OBTAINED FROM LONG TRANSMISSION SCANS.

\begin{tabular}{l|l|l}
\hline Segment & CT (units) & $\mu(1 / \mathrm{cm})$ \\
\hline Background & 424 & 0.0 \\
\hline Soft tissue & 1434 & 0.056 \\
\hline Spongiosa Bone & 2506 & 0.066 \\
\hline Cortical Bone & 5457 & 0.100 \\
\hline
\end{tabular}

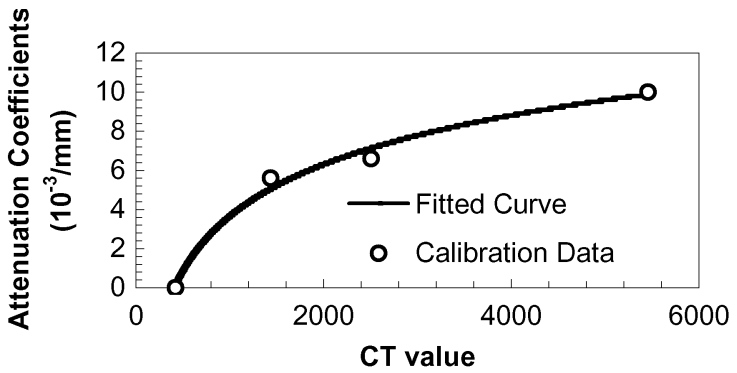

Fig. 3. $\mu$ and CT values of the attenuation phantom in Table I were fit with a Log square function to derive an empirical relationship.

Inc., Knoxville, TN) were obtained for the attenuation calibration phantom. A transmission $\mu$ image was reconstructed from these data sets by filtered backprojection [6].

The CT image of the phantom was first segmented so that the attenuation-equivalent materials were assigned to one category or one segment. The CT image was registered to that of the PET transmission image. The voxels in each segment of the transmission image were averaged to give a $\mu$ for the material belonging to that segment. The CT value for a segment is the segment peak location in its histogram. Table I shows the raw CT values and the corresponding $\mu$ values. Note that Hounsfield units could be a middle step for converting raw CT data to $\mu$ for PET; however, it was not a necessary step and was skipped in this paper.

Fitting the CT and $\mu$ values in Table I with a Log square function

$$
\mu=a_{0}+a_{1} \log _{10} \mathrm{CT}+a_{2}\left(\log _{10} \mathrm{CT}\right)^{2}
$$

gives the curve shown in Fig. 3. The fitted function was then used to convert CT images into $\mu$ maps for PET. This calibration strategy was practical because 1) only raw CT data were available on the CT system and 2) scatter correction was not yet implemented for the emission data; therefore, theoretical $\mu$ values could not be used directly.

\section{F. Analytic $\mu$ Map}

Several operations were required to obtain the analytic $\mu$ map. The PET emission data were first segmented to extract the outline of the animal's body. Since the histogram of the original emission image was heavily weighed toward the background (low counts) side, the thresholding algorithm had difficulty identifying the body outline. Accordingly, a more equalized histogram was obtained by first applying the logarithmic function to the emission image followed by the segmentation method of $\mathrm{Xu}$ et al. [7] developed for human PET transmission images. After segmenting the background and the body, the body region was filled with an empirical $\mu$ to represent the animal's body $\mu$
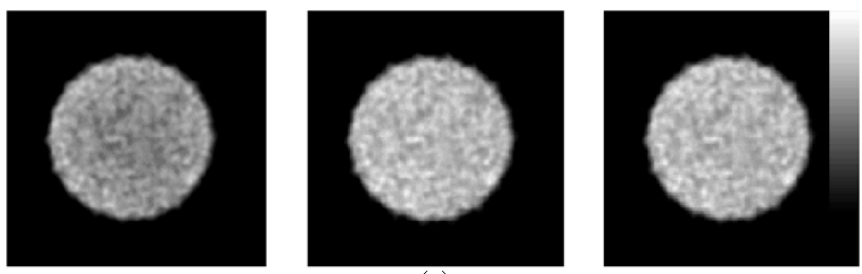

(a)

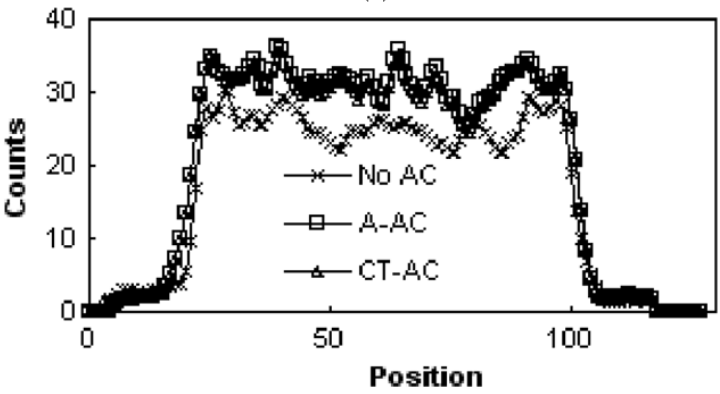

(b)

Fig. 4. (a) (Top): Images of a transverse slice of the uniform cylinder phantom (left) without AC, (center) with AC, and (right) with CT-AC. The darker central area in the left image is compensated in the $\mathrm{AC}$ applied center and right images. (b) (Bottom): Horizontal profiles passing near the center of the cylinder phantom images shown in (a). Both A-AC and CT-AC methods restored the central area activity. The difference between the two methods is small.

map. The single empirical $\mu$ value was obtained by averaging the corresponding CT-derived $\mu$ map. A $\mu$ map for the bed only was separated from the corresponding CT-derived $\mu$ image and later added to the body $\mu$ map to get a final $\mu$ map for AC. In an actual implementation where no CT data is available, the CT-derived bed $\mu$ map can be replaced by a fixed bed-shaped structure filled with an assigned $\mu$ value. The vertical position of this superimposed bed-shaped structure relative to the position of the emission contour can be inferred from an independent measurement of the vertical position of the actual bed during the scan.

\section{G. Implementation of Attenuation Correction}

The $\mu$ maps generated by both methods were then forwardprojected using the forward projection routine in the 3D reconstruction program to obtain the attenuation factors. The attenuation factors were then incorporated in the 3D reconstruction as a multiplication term contributing to the system matrix of the expectation maximization (EM) algorithm. For each study, the number of iterations and subsets were kept fixed for different $\mathrm{AC}$ schemes on any one subject including no correction.

\section{RESULTS}

Images of a transverse slice of the cylinder phantom without attenuation corrections (No AC), with attenuation corrections (A-AC), and with CT attenuation corrections (CT-AC) are shown in Fig. 4(a). The profiles passing through a point near the center of the cylinder are shown in Fig. 4(b). The restoration of the two AC methods is apparent compared to no correction. For this uniform cylinder, the A-AC method using an empirical effective attenuation coefficient that includes scatter compared favorably to the CT-AC method.

Table II reports the mean and coefficient of variation (COV) of activity in the uniform cylinder phantom without $\mathrm{AC}$, with $\mathrm{A}-\mathrm{AC}$, and with CT-ACs. To make these measurements, nine 
TABLE II

EMISSION CONCENTRATION (ARBITRARY UNITS) IN CYLINDER PHANTOM WITH AND WITHOUT ATTENUATION CORRECTIONS

\begin{tabular}{l|l|l}
\hline Attn. Corr. & Mean & COV $\left(\times 10^{-3}\right)$ \\
\hline None & 26.38 & 6.58 \\
\hline Analytic & 31.31 & 6.70 \\
\hline CT & 30.93 & 6.92 \\
\hline
\end{tabular}
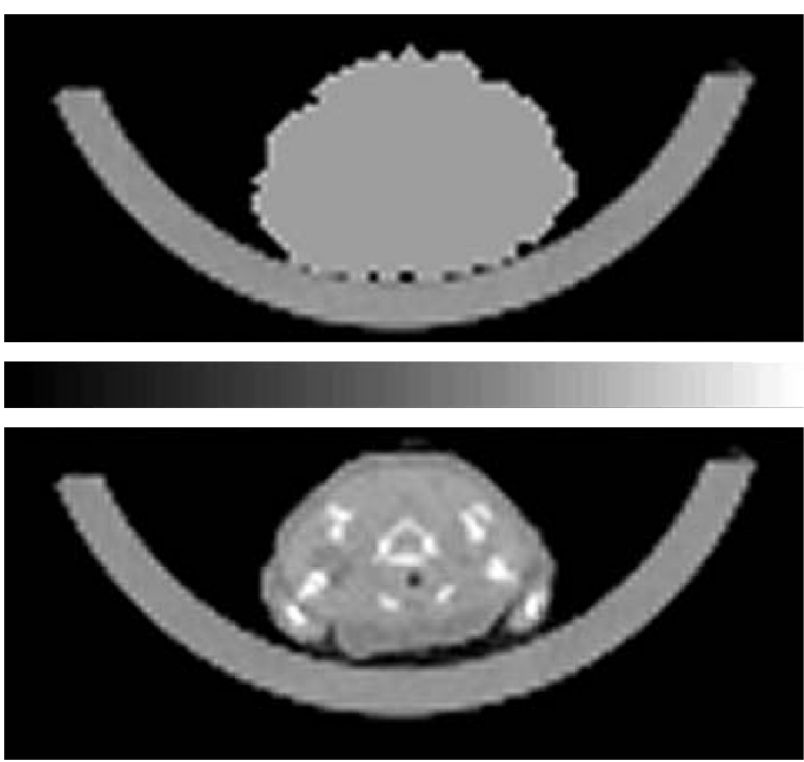

Fig. 5. $\mu$ image obtained with the (top) analytic method and (bottom) CT method of a transverse slice near the heart of the mouse. The color palette (middle) is used for both images. The outline of the analytically derived map resembles that of the CT image but is slightly larger.

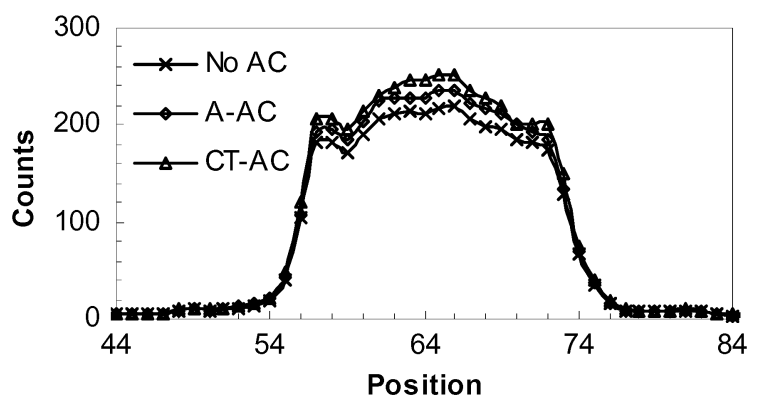

Fig. 6. Section profiles across the fillable cavity (cylinder D in Fig. 1) of the attenuation calibration phantom without $\mathrm{AC}$, with $\mathrm{A}-\mathrm{AC}$, and with $\mathrm{CT}-\mathrm{AC}$ applied to the emission data.

circular regions of interest (ROIs), each $40 \mathrm{~mm}$ in diameter, were defined on each of the nine axially centered slices. The nine middle slices were chosen to avoid the axial sensitivity variation at the ends of the FOV. The analytic method showed somewhat less noise and good restoration of the central activity for this uniform and geometrically simple object.

Fig. 5 shows analytically and CT-derived $\mu$ maps of a transverse slice near the heart of the mouse.

The section profiles across the fillable cavity (chamber D in Fig. 1) containing F-18 in the attenuation phantom without AC, with A-AC, and with CT-AC are shown in Fig. 6. Since the cavity is only a small fraction of the full phantom and the rest does not have any positron emitting materials in it, the analytically derived $\mu$ inevitably underestimated the true attenuation
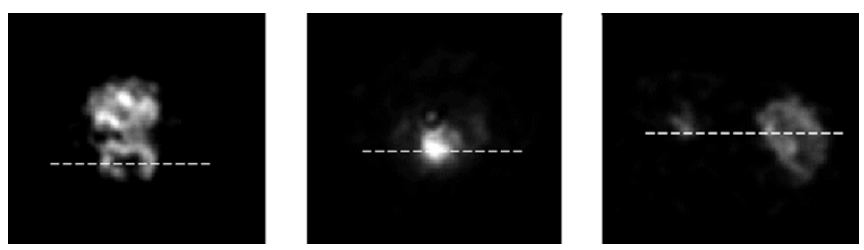

(a)

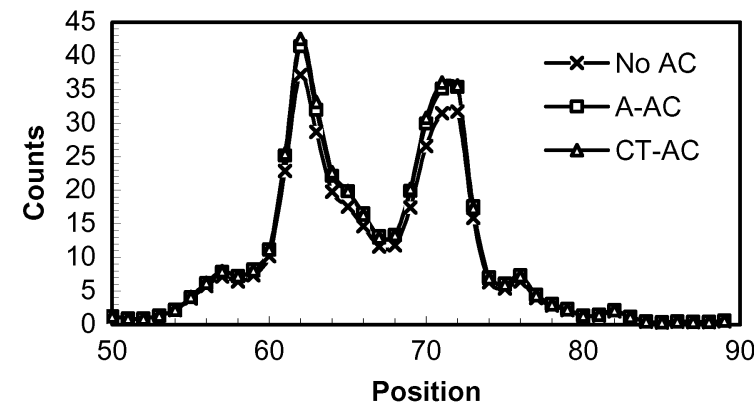

(b)

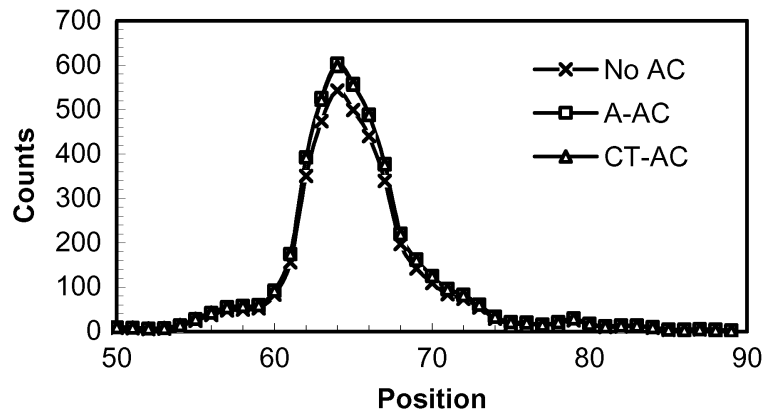

(c)

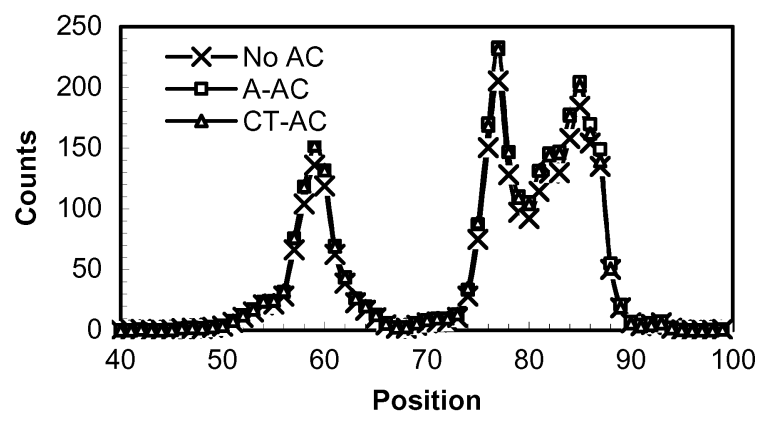

(d)

Fig. 7(a). Head (left), heart (center), and tumor (right) transverse images as indicated by the top, middle, and bottom short horizontal lines in the sagittal view, respectively, in Fig. 2. The dashed line in each of the transverse images identifies the location where the horizontal profile was generated for Figs. 7(b), (c), and (d), respectively. (b) Horizontal profiles without AC, with analytic AC (A-AC), and with CT-AC), for the head slice in 6(a) (left). (c) Horizontal profiles without $\mathrm{AC}$, with analytic (A-AC), and with CT-AC for the heart slice in 6(a) (center). (d) Horizontal profile without $\mathrm{AC}$, with analytic (A-AC), and with CT-AC for tumor slice in 6(a) (right).

and the corrected image has lower intensity, compared with the CT method. The elevated central parts of the profiles are likely due to the partial volume effect that significantly underestimates emission activity at the edges of the cylindrical chamber. The diameter of this cylinder is only $1 \mathrm{~cm}$ and when imaged with a 2 $\mathrm{mm}$ resolution scanner would likely yield these profiles.

Fig. 7 compares the performance of the analytic and CT attenuation correction methods by showing the profiles at selected locations in three slices: one in the head, one across the heart, 
and one across the thigh of the mouse. The corresponding transverse section images and the points where the profiles were extracted are illustrated in Fig. 7(a). The three profiles with no AC, with A-AC, and with CT-AC are compared in Fig. 7(b)(c), and (d), respectively.

\section{DiscuSSION AND CONLCUSION}

Our results show that the computed tomography with attenuation correction (CT-AC) method achieved activity intensity restoration in all three data sets and contrast recovery in the attenuation calibration phantom and the mouse study. Knowing that CT provides high resolution attenuation images of the imaging subject, this is expected. The results confirm that the implementation was correct.

The analytic attenuation correction (A-AC) method was insufficient for the attenuation phantom as a result of segmentation missing most of the nonemission source areas. For the uniform cylinder phantom and the mouse study, the analytic method showed similar correction factors compared to the CT-AC method. The likely reason for this is that high or low density regions in the mouse, such as bone and air-filled cavities, comprise only a small fraction of the soft-tissue body cross section of the mouse, and the dimensions of these heterogeneous regions are typically small. In addition, the lungs of the dead mouse were collapsed and were only slightly less dense than soft tissue, making the torso of the mouse appear relatively uniform. This observation reminds that CT and positron emission tomography (PET) scans should be made with the animal in the same state, dead or alive, to have matching conditions for attenuation correction. These data suggest, therefore, that the A-AC method may be the method of choice, compared to CT-AC, when imaging a target with reasonably uniform composition. For imaging regions of live small animals that contain heterogeneous tissue distributions, e.g., the thorax, more sophisticated corrections may be necessary.

The curve fitting method for deriving the relationship between $\mu$ for PET and CT-values is based on a given phantom size. However, the effective attenuation coefficient $\mu_{\text {eq }}$ depends on the amount of scatter that may itself vary with the size of the animal. As a result, more than one calibration curve might be needed depending on animal size. To estimate the dependence of $\mu_{\mathrm{eq}}$ on object size, we measured the scatter fractions for two uniform cylinder phantoms with diameters of $3 \mathrm{~cm}$ and $5 \mathrm{~cm}$ to be $14 \%$ and $23 \%$, respectively. The $\mu_{\mathrm{eq}}$ values calculated for these two phantoms were very close to one another, 0.046 and $0.0441 / \mathrm{cm}$, respectively, suggesting that scatter and attenuation partially offset one another as object size increases and that a single value may suffice for objects in this size range.

It is also noteworthy that reasonable agreement exists between the corrected and uncorrected count profiles shown in Fig. 7(a)-(d). This observation implies that attenuation in mice is a second-order effect and so can be corrected by an approximate $\mathrm{A}-\mathrm{AC}$ method that might fail in larger subjects. In this case, the $\mathrm{A}-\mathrm{AC}$ method is making a correction to an already small error so the fundamental accuracy of the correction need not be very high.

\section{ACKNOWLEDGMENT}

The work in this paper utilized the high-performance computational capabilities of the SGI PentiumIII/Linux Cluster at the Center for Computational Research, State University of New York at Buffalo. The authors would like to thank Dr. C. Johnson for providing the three-dimensional reconstruction code and $\mathrm{S}$. English from the Radiation Biology Branch of NCI for supplying the mouse.

\section{REFERENCES}

[1] J. Seidel, J. J. Vaquero, and M. V. Green, "Resolution uniformity and sensitivity of the NIH ATLAS small animal PET scanner: Comparison to simulated LSO scanners without depth-of-interaction capability," IEEE Trans. Nucl. Sci., vol. 50, no. 5, pp. 1347-1350, Oct. 2003.

[2] P. E. Kinahan, D. W. Townsend, T. Beyer, and D. Sashin, "Attenuation correction for a combined 3D PET/CT scanner," Med. Phys., vol. 25, pp. 2046-2053, 1998.

[3] P. L. Chow, R. Rannou, and A. Chatziioannou, "Attenuation correction for a 3D small animal PET tomograph, using X-ray microCT," Mol. Imag. Biol., vol. 4, pp. S17-S18, 2002.

[4] M. J. Paulus, S. S. Gleason, S. J. Kennel, P. R. Hunsicker, and D. K. Johnson, "High resolution x-ray computed tomography: An emerging tool for small animal cancer research," Neoplasia, vol. 2, pp. 62-70, 2000.

[5] C. A. Johnson, J. Seidel, J. J. Vaquero, J. Pascau, M. Desco, and M. V. Green, "Exact positioning for OSEM reconstructions on the ATLAS depth-of-interaction small animal scanner," Mol. Imag. Biol., vol. 4, p. S22, 2002.

[6] J. A. Fessler, "ASPIRE 3.0 user's guide: A sparse iterative reconstruction library," Communications and Signal Processing Laboratory, Dept. of EECS, Univ. of Michigan, Ann Arbor, 1995.

[7] M. Xu, P. D. Cutler, and W. K. Luk, "Adaptive, segmented attenuation correction for whole-body PET imaging," IEEE Trans. Nucl. Sci., vol. 43, no. 1, pp. 331-336, Feb. 1996. 